\title{
Adaptive tracking control for uncertain nonlinear systems subject to unknown control coefficients, state constraints, and input saturation
}

\author{
Chunxiao Wang ${ }^{1},{ }^{*} \quad \mathrm{Lu}^{\mathrm{Qi}}{ }^{1}, \quad \mathrm{Yan} \mathrm{Zhao}^{1}, \quad$ and $\mathrm{Jiali} \mathrm{Yu}^{2}$ \\ 1.School of Science, Shandong Jianzhu University, Ji'nan, 250101, China \\ 2.School of Mathematical Sciences, University of Electronic Science and Technology of China, \\ Chengdu, 610054, China
}

\begin{abstract}
This article is committed to studying the tracking control problem for a class of uncertain nonlinear system with unknown control coefficients. The system is subject to full state constraints, input saturation constraint, and external disturbances simultaneously. By introducing a hyperbolic tangent function to approximate the saturated input function, the sharp corner caused by the input saturation is avoided. In the meanwhile, an auxiliary system is constructed to compensate the resulting approximation error. By using the barrier Lyapunov function (BLF) based adaptive backsteping control, the Nussbaum-type adaptive controllers are constructed for the augmented system with unknown control direction. It not only ensures the system states are always within the constrained range, but also guarantees the tracking performance of the system, no matter whether the control direction of the system is known or not. Meanwhile, dynamic surface control (DSC) is used in the controller design, which avoids "computation explosion" caused by the repeated derivation of virtual control law. Aiming at the nonparametric uncertainty of the system, a common adaptive law is designed by combining the unknown constant bounds of the external disturbance with the error term caused by input saturation estimation. It improves the tracking performance of the system and reduces the burden of the controller greatly. Finally, a simulation example is given to demonstrate the effectiveness of the proposed control scheme in three scenarios.
\end{abstract}

Keywords: Barrier Lyapunov function, input saturation constraint, Nussbaum gain function, state constraint, unknown control direction.

\section{Introduction}

In the past few decades, the tracking control problem for uncertain nonlinear system has attracted great public attention due to its wide applications[1,2,3]. Self-driving cars and ships, for example, follow the trajectory set by their navigation systems[4]. The tracking performance is directly related to the safety and efficiency of automatic driving. System uncertainties are important factors leading to system instability, poor tracking performance or other undesirable consequence $[5,6]$. In a broad sense, system uncertainty mainly can be classified into parametric uncertainty and nonparametric uncertainty. It is well known that the adaptive backstepping control is a classical control methods for parametric nonlinear system[1,7]. When there are nonparametric uncertainties in nonlinear system, the unknown dynamics can be approximated by neural networks or fuzzy systems $[8,9,10]$. Although great achievements have been made in the tracking control of uncertain nonlinear systems, it is worth noting that all the mentioned results are based on the fact that the control coefficients of the system are known, or at least the control directions (the signs of the control coefficients) are available.

The control coefficients or called control gains, which represent the control directions are usually unknown, especially in practical systems[11, 12]. For instance, a priori knowledge of the control direction of a

${ }^{*}$ Corresponding author. Email: xiao2166@126.com 
ship steering system is usually unavailable due to the real-time variations of speed, changes in loading condition, and various complicated environmental factors [12]. When there is no available knowledge about the control gain, all existing control methods for systems with known control coefficients are no longer applicable, and the control problem of nonlinear systems becomes very difficult. In 1983, Nussbaum put forward the Nussbaum-gain technology, which solved the controller design problem of nonlinear system with unknown signs of control coefficients [13]. Based on this, a great deal of works have been done by scholars in recent years, which greatly promote the development of nonlinear systems with unknown control direction both in theory and application $[14,15,16,17,18]$. By using the Nussbaum gain technology, article [14] investigated the adaptive tracking control problem for a class of strict-feedback nonlinear systems with unknown control direction. A Nussbaum gain function-based quantized feedback control was proposed for nonlinear feedforward systems with both unknown control coefficients and unknown output functions in article [15]. In [16], Sun et al. proposed a novel Nussbaum-type function, based on which an adaptive fuzzy asymptotically tracking control scheme was designed for full state constrained nonlinear system with unknown control direction.

Despite plentiful advancements mentioned above, there is still much research room for the nonlinear system with unknown control direction. Most of the above referred researches are aimed at the control of the unknown control gain nonlinear systems with free state, output and input. However, constraints are ubiquitous and crucial in practical as it is relevant to the physical limits and security performance of system [19, 20]. For example, the input amplitude of the rudder of ships is not allowed to be infinitely large, which is an input saturation constraint problem[4]. If it is not taken into account in the ship course tracking control design, it will reduce its tracking performance and even bring unexpected risks. In order to avoid the movable electrode of the electrostatic microactuator contact with the fixed electrodes during operation, its trajectory is constrained within a fixed range, which is a state constraint problem [19]. State/output constraint and input constraint are the two most common types of constraints, which received considerable attentions in recent years. Various methods for dealing with state/output constraints have sprung up, such as reference governors control [21], positive invariant sets [22], model predictive control [23], barrier Lyapunov function (BLF) [24, 25, 26, 27, 28], and state transformation $[29,30,31]$, etc. BLF is the most popular technique to deal with state/output constraint in recent years[24, 25, 26, 27, 28]. By designing a control to make the derivative of BLF negative semidefinite, which avoids the need for explicit solution of the system. On the other hand, input constraint subject to actuator saturation has also received increasing attention[32, 33, 34, 35, 36]. Among them, the input saturation constraint was compensated by using neural networks (NN) [32], reference governors [33], and dynamic inversion model reference control [34], respectively. Recently, the hyperbolic tangent function was used to approximate the saturated input function, and then compensate for the introduced error by an auxiliary system $[8,35,36]$. Nevertheless, to the best of the authors' knowledge, all these above mentioned works either do not take into account the unknown control direction of the system, or ignore the effect of the state/output constraint and input constraint for the system stability, which motivates this paper.

Motivated by the above observations, in this article, we are committed to developing a tracking control scheme for the state and input constrained uncertain nonlinear systems with unknown control coefficients. The main contributions are summarized as follows:

(1) This paper studies a class of nonlinear systems with full state constraint, input saturation constraint, external disturbances, and unknown control coefficients. On the one hand, a hyperbolic tangent function is used to approximate the saturation input function, and then an auxiliary system is constructed to compensate 
the introduced error, which solves the input saturation constraint problem of the system well. On the other hand, a BLF-based adaptive controller is constructed for the augmented system, which ensures the stability of the system and averts the violation of state constraints for all the system running time. Compared with our control scheme, the nonlinear uncertainty in $[8,36]$ is approximated by neural network, which leading to a heavy computational burden as the nodes of $\mathrm{NN}$ increase.

(2) Compared with the existed results $[8,36]$, the considered system of this paper is more general, where the signs of control gains are not required to be with a prior knowledge. To facilitate the controller design, Nussbaum gain functions are utilized to solve the difficulty caused by unknown control coefficient.

(3) The adaptive law is designed by combining the unknown constant boundary of the system external disturbance with the error term caused by the input saturation estimation. By minimizing the design parameters, the burden of the controller is reduced and the tracking performance of the system is improved. Additionally, dynamic surface control is adopted which solved the problem of computation explosion caused by repeated derivation of virtual control law.

The organization of the rest of this article is as follows. Section 2 describes the statement of the problem and some necessary definitions, assumptions, and lemmas are given. Section 3 shows the explicit controller design procedure and stability analysis. Meanwhile, the main results of this article are summarized in a theorem. Following, a simulation example is given in Section 4 to illustrate the effectiveness of the presented controller. Finally, Section 5 ends this paper with the conclusions.

\section{Preliminaries}

Consider the following semi-strict feedback nonlinear systems

$$
\left\{\begin{aligned}
\dot{x}_{j}(t) & =\theta_{j} \psi_{j}\left(\bar{x}_{j}(t)\right)+g_{j}\left(\bar{x}_{j}(t)\right) x_{j+1}(t)+D_{j}(x, t), \\
(j & =1,2, \cdots, n-1) \\
\dot{x}_{n}(t) & =\theta_{n} \psi_{n}\left(\bar{x}_{n}(t)\right)+g_{n}\left(\bar{x}_{n}(t)\right) u(v)+D_{n}(x, t) \\
y(t) & =x_{1}(t)
\end{aligned}\right.
$$

where $\bar{x}_{j}(t)=\left(x_{1}(t), \cdots, x_{j}(t)\right)^{T} \in \mathbb{R}^{j}(j=1,2, \cdots, n)$ and $y(t) \in \mathbb{R}$ are the system states and output, respectively; $g_{j}(\cdot): \mathbb{R}^{j} \rightarrow \mathbb{R}$ is unknown smooth control gain function, $\theta_{j}$ is unknown constant parameter with known boundry; $\psi_{j}(\cdot): \mathbb{R}^{j} \rightarrow \mathbb{R}$ is known continuous function; $D_{j}(x, t): \mathbb{R}^{n} \times \mathbb{R}^{+} \rightarrow \mathbb{R}$ represents the unknown external disturbance; $u(v(t))$ is the input saturation function, it is obtained by passing the control input signal $v(t)$ through a saturation constraint $\bar{u}$, which described by

$$
u(v(t))=\operatorname{sat}(v(t))= \begin{cases}\operatorname{sign}(v(t)) \bar{u}, & |v(t)| \geq \bar{u} \\ v(t), & |v(t)|<\bar{u}\end{cases}
$$

where $\bar{u}$ is a known input saturation constant bound. Obviously, there is a sharp corner between $u(v(t))$ and $v(t)$ when $|v(t)|=\bar{u}$. By referring to $[8,36]$, the following smooth function can be used to approximate the input saturation function:

$$
\hbar(v)=\bar{u} \times \tanh \left(\frac{v}{\bar{u}}\right)=\bar{u} \cdot \frac{e^{\frac{v}{\bar{u}}}-e^{-\frac{v}{\bar{u}}}}{e^{\frac{v}{\bar{u}}}+e^{-\frac{v}{\bar{u}}}} .
$$


Then, the saturation function $\operatorname{sat}(v(t))$ can be expressed as

$$
\operatorname{sat}(v)=\hbar(v)+\rho(v)=\bar{u} \times \tanh \left(\frac{v}{\bar{u}}\right)+\rho(v),
$$

where $\rho(v)$ is the approximation error. Obviously, it is bounded, which can be seen from the detailed expression below

$$
|\rho(v)|=|\operatorname{sat}(v)-\hbar(v)| \leq \bar{u}(1-\tanh (1))=\bar{\rho} .
$$

On the other hand, it is worth mentioning that the system states are measurable and subject to constraints: $\Omega_{x_{j}}:=\left\{x_{j}|| x_{j}(t) \mid<k_{c_{j}}\right\}(j=1, \cdots, n)$, where $k_{c_{j}}$ are positive constants. They are required to remain in the predefined constrained regions for all the system running time.

To handle the input saturation constraint, with the help of equations (3)-(5), we use the neural network approximation to augment the $n$-order system (1) to the following $(n+1)$-order system,

$$
\left\{\begin{array}{l}
\dot{x}_{j}(t)=\theta_{j} \psi_{j}\left(\bar{x}_{j}(t)\right)+g_{j}\left(\bar{x}_{j}(t)\right) x_{j+1}(t)+D_{j}(x, t), \\
\quad(j=1,2, \cdots, n-1) \\
\dot{x}_{n}(t)=\theta_{n} \psi_{n}\left(\bar{x}_{n}(t)\right)+g_{n}\left(\bar{x}_{n}(t)\right) \hbar(v)+\bar{D}_{n}(x, t), \\
\dot{v}(t)=\mu-\zeta v(t), \\
y(t)=x_{1}(t)
\end{array}\right.
$$

where $v$ and $\mu$ are the auxiliary state and control signals, $\zeta$ is a positive constant, $\bar{D}_{n}(x, t)=D_{n}(x, t)+$ $g_{n}\left(\bar{x}_{n}(t)\right) \rho(v)$.

Remark 1. By introducing the hyperbolic tangent function to approximate the saturated input function, the problem of the sharp corner caused by the input function is avoided. Further more, the auxiliary system (6) is constructed to compensate the resulting approximation error. In system (6), the term $\bar{D}_{n}(x, t)$ is the combination of the unknown constant boundary of the system external disturbance and the upper bound of the input saturation approximation error. We combine these uncertainties together and design a common adaptive law, which greatly reduces the burden of the controller.

The control aim of this article is to design a state feedback control strategy for the system (1), which drives the system output $y$ to track a reference trajectory $y_{d}(t)$ as close as possible. In the meanwhile, all state constraints should be guaranteed during the system running time. To achieve the control goal, the following assumptions, definition and lemmas are necessary and practical.

Assumption 1. $\theta_{j}(j=1, \cdots, n)$ in system (1) represent the unknown parametric uncertainties with known boundry. Suppose that there exist a positive constant $\theta_{M}$ such that $\left|\theta_{j}\right| \leq \theta_{M}$. On the other hand, $D_{j}(x, t)(j=$ $1, \cdots, n)$ in system (1) denote the external disturbances, which is bounded. Suppose that there exist unknown positive constants $d_{j}$ such that $\left|D_{j}(x, t)\right| \leq d_{j}$.

Assumption 2. The functions $g_{j}(\cdot)(j=1, \cdots, n)$ are unknown but bounded. Suppose that there exist positive constants $\bar{g}_{j}$ such that $0<\left|g_{j}(\cdot)\right| \leq \bar{g}_{j}$ for $\forall x_{j} \in \Omega_{x_{j}}$.

Assumption 3. The reference signal $y_{d}(t)$ is smooth and available, which satisfies

$$
\left[y_{d}, \dot{y}_{d}, \ddot{y}_{d}\right]^{T} \in \Omega_{0}=\left\{\left[y_{d}, \dot{y}_{d}, \ddot{y}_{d}\right]^{T}: y_{d}+\dot{y}_{d}+\ddot{y}_{d} \leq \omega\right\},
$$

where $\omega$ is a constant. 
Remark 2. The above assumptions are standard and necessary. Assumptions 1 is the standard assumption for semi-strict feedback nonlinear system, and the constant bounds for $\theta_{j}$ and $D_{j}(x, t)$ are necessary and reasonable in adaptive backstepping tracking control. Assumptions 2 indicates that the control direction of system (1) is unknown but boundedness, which is not only reasonable and necessary but also widely available in many important practical systems, such as high speed trains, ship steering system, and aerial vehicles, etc. Assumptions 3 is also the standard assumption in adaptive backstepping tracking control.

Remark 3. Compared with the system studied in $[8,36]$, the considered system of this paper is more general. Assumptions 2 indicates that the control gains $g_{i}$ of system (1) are unknown, while the control gains of the system in articles $[8,36]$ are known constants. So the systems discussed in $[8,36]$ are our special case. Additionally, state constraints are not considered in [36].

Definition $\mathbf{1}^{[13]}$. If the following properties hold for any continuous function $N(\zeta)$,

$$
\begin{aligned}
& \lim _{\iota \rightarrow \infty} \sup \frac{1}{\iota} \int_{0}^{\iota} N(\zeta) d \zeta=+\infty \\
& \lim _{\iota \rightarrow \infty} \inf \frac{1}{\iota} \int_{0}^{\iota} N(\zeta) d \zeta=-\infty
\end{aligned}
$$

then $N(\zeta)$ is called a Nussbaum-type function. Such as $\exp \left(\zeta^{2}\right) \cos \left(\frac{\pi}{2} \zeta\right), \zeta^{2} \cos (\zeta)$ and $\zeta^{2} \sin (\zeta)$ are all familiar Nussbaum-type functions. In this paper, the Nussbaum-type function is selected as $N(\zeta)=\zeta^{2} \cos (\zeta)$.

Lemma $1^{[28]}$. Given $U \in \mathbb{R}$, there exist a hyperbolic function $\tanh (\cdot)$ such that the following inequality holds for any $\varepsilon>0$,

$$
0 \leq|U|-U \tanh \left(\frac{U}{\varepsilon}\right) \leq k_{\epsilon} \varepsilon
$$

where $k_{\epsilon}=e^{-\left(k_{\epsilon}+1\right)}$, ie, $k_{\epsilon}=0.2785$.

Lemma $2^{[14,17]}$. Let $V(\cdot)$ and $\xi(\cdot)$ be smooth functions defined on $\left[0, t_{f}\right)$ and $V(\cdot) \geq 0, N(\xi)$ is a smooth even Nussbaum-type function. For $\forall t \in\left[0, t_{f}\right)$, if there exist positive constants $c_{0}$ and $c_{1}$, such that

$$
V(t) \leq c_{0}+e^{-c_{1} t} \int_{0}^{t}(g(\tau) N(\zeta(\tau))+1) \dot{\zeta}(\tau) e^{c_{1} \tau} d \tau
$$

where $g(\tau) \neq 0$ is a bounded time-varying function, then $V(t), \zeta(t)$ and $\int_{0}^{t} g(\cdot) N(\zeta) \dot{\zeta} d \tau$ are all bounded on $\left[0, t_{f}\right)$.

Remark 4. Lemma 2 is developed by basing on the definition of Nussbaum function, which is always used to deal with the issue of uncertain control gain. What's more, according to [17, 18], if the solution of closed-loop system is bounded on $\left[0, t_{f}\right)$ for any given $t_{f}>0$, then $t_{f}=\infty$.

\section{Robust Adaptive controller design and stability analysis}

In this section, we design the robust adaptive tracking controller for the expanded system (6) based on the backstepping control. A first order dynamic surface is established to avoid the repeated derivation for the virtual controllers, and the BLF technique is utilized to avert the violation of state constraints. From this point onwards, for the sake of convenience in writing and structure clear, if there are no confusion arise, some arguments of the functions will be omitted. 


\subsection{Preliminaries}

To achieve the predetermined control objective, we first do the following coordinate transformations:

$$
\begin{aligned}
& S_{1}=x_{1}-y_{d}, \\
& S_{j}=x_{j}-\alpha_{j f},(j=2, \cdots, n), \\
& S_{n+1}=\hbar(v)-\alpha_{(n+1) f}, \\
& Z_{j}=\alpha_{j f}-\alpha_{j-1},(j=2, \cdots, n+1),
\end{aligned}
$$

where $S_{j}(j=1, \cdots, n+1)$ denote the actual/virtual tracking errors; $\alpha_{j}(j=2, \cdots, n+1)$ represent the virtual control laws; $\alpha_{j f}(j=2, \cdots, n+1)$ are intermediate variables which are produced by the following first-order filters,

$$
\tau_{j} \dot{\alpha}_{j f}+\alpha_{j f}=\alpha_{j-1}, j=2, \cdots, n+1
$$

where $\tau_{j}$ are time constants; $Z_{j}$ denote the filter surface errors.

Since $\theta_{j}$ and $d_{j}(j=1, \cdots, n)$ are parametric uncertainties and unknown external disturbance bounds, respectively, we define variables $\hat{\theta}_{j}$ and $\hat{d}_{j}$ to estimate them. The estimation errors are denoted as follows

$$
\begin{gathered}
\tilde{\theta}_{j}=\hat{\theta}_{j}-\theta_{j}, \\
\tilde{d}_{j}=\hat{d}_{j}-d_{j} .
\end{gathered}
$$

For dealing with the full state constraints, the following tan-type BLFs are introduced [28, ?]:

$$
V_{j}^{*}=\frac{k_{b_{j}}^{2}}{\pi} \tan \left(\frac{\pi S_{j}^{2}}{2 k_{b_{j}}^{2}}\right),\left|S_{j}(0)\right|<k_{b_{j}}, j=1, \cdots, n,
$$

where $k_{b_{j}}$ are positive constants. Computing the time derivative of $V_{j}^{*}$, it follows that

$$
\dot{V}_{j}^{*}=\vartheta_{s_{j}} \dot{S}_{j}
$$

where $\vartheta_{s_{j}}=\frac{S_{j}}{\cos ^{2}\left(\frac{\pi S_{j}^{2}}{2 k_{b_{j}}^{2}}\right)}$.

Remark 5. Obviously, the tan-type BLF (15) restrains $S_{j}$ within the region $\Omega_{s_{j}}:=\left\{S_{j} \in \mathbb{R}|| S_{j} \mid<k_{b_{j}}\right\}$ $(j=1, \cdots, n)$ for any $t \geq 0$ as long as $\left|S_{j}(0)\right|<k_{b_{j}}$. It is through the bounds of $S_{j}$ that we ensure the constraint satisfaction of $x_{j}$. The specific proving process will be given in the proof of the theorem later. What's more, compared with the most commonly used log-type BLF for symmetric state constraints [25, 27, 37], the tan-type BLF has significant advantages. When there are no constraints on system states, that is $k_{c_{j}} \rightarrow \infty$, hence $k_{b_{j}} \rightarrow \infty, j=1, \cdots, n$. Using L'Hospital rule we have

$$
\lim _{k_{b_{j}} \rightarrow \infty} \frac{k_{b_{j}}^{2}}{\pi} \tan \left(\frac{\pi S_{j}^{2}}{2 k_{b_{j}}^{2}}\right)=\frac{1}{2} S_{j}^{2} .
$$

If some states in the system are constrained and some are free, we can design a unified tan-type BLF without the need for separate case discussion as in [37]. Hence, the tan-type BLF is advantageous to integrate the constraint analysis into a unified algorithm. 


\subsection{Robust adaptive controller design}

In this subsection, we present the detailed adaptive backstepping design procedure step by step for system (6), which is essential to achieve our control goal.

Step 1. Starting with the $x_{1}$-subsystem of system (6), differentiating $S_{1}$ with respect to time and invoking equations (6), (8) and (9), it yields

$$
\dot{S}_{1}=\dot{x}_{1}-\dot{y}_{d}=\theta_{1} \psi_{1}+g_{1}\left(S_{2}+\alpha_{2 f}\right)+D_{1}-\dot{y}_{d} .
$$

To deal with the system state constraint, parametric uncertainty, and external disturbance simultaneously, the following Lyapunov candidate function is constructed

$$
V_{1}=V_{1}^{*}+\frac{1}{2 \lambda_{\theta_{1}}} \tilde{\theta}_{1}^{2}+\frac{1}{2 \lambda_{d_{1}}} \tilde{d}_{1}^{2}+\frac{1}{2} Z_{2}^{2}
$$

where $\lambda_{\theta_{1}}$ and $\lambda_{d_{1}}$ are positive constants. Base on the equations (11), (16) and (18), the time derivative of $V_{1}^{*}$ can be rewritten as

$$
\dot{V}_{1}^{*}=\vartheta_{s_{1}} \cdot\left(\theta_{1} \psi_{1}+g_{1}\left(S_{2}+Z_{2}+\alpha_{1}\right)+D_{1}-\dot{y}_{d}\right) .
$$

Utilizing Assumption 1 and Lemma 1, it follows that

$$
\vartheta_{s_{1}} D_{1} \leq\left|\vartheta_{s_{1}}\right| d_{1} \leq d_{1}\left(k_{\epsilon} \varepsilon+\vartheta_{s_{1}} \tanh \left(\frac{\vartheta_{s_{1}}}{\varepsilon}\right)\right),
$$

where $\varepsilon$ is a positive parameter, $k_{\epsilon}=0.2785$. In the meanwhile, involving the Young's inequality, it follows that

$$
\begin{aligned}
g_{1} \vartheta_{s_{1}} S_{2} & \leq \frac{l_{1}}{2} \vartheta_{s_{1}}^{2}+\frac{\bar{g}_{1}^{2}}{2 l_{1}} S_{2}^{2}, \\
g_{1} \vartheta_{s_{1}} Z_{2} & \leq \frac{l_{1}}{2} \vartheta_{s_{1}}^{2}+\frac{\bar{g}_{1}^{2}}{2 l_{1}} Z_{2}^{2},
\end{aligned}
$$

where $l_{1}$ is a positive parameter, $\bar{g}_{1}$ is the upper bound of $\left|g_{1}\right|$. Then, computing the time derivative of $V_{1}$ based on (13), (14) and (19)-(23), it yields

$$
\begin{aligned}
\dot{V}_{1} \leq & \vartheta_{s_{1}} \cdot\left(\theta_{1} \psi_{1}+g_{1} \alpha_{1}-\dot{y}_{d}\right)+l_{1} \vartheta_{s_{1}}^{2}+\frac{\bar{g}_{1}^{2}}{2 l_{1}} S_{2}^{2}+\frac{\bar{g}_{1}^{2}}{2 l_{1}} Z_{2}^{2} \\
& +d_{1}\left(k_{\epsilon} \varepsilon+\vartheta_{s_{1}} \tanh \left(\frac{\vartheta_{s_{1}}}{\varepsilon}\right)\right)+\frac{1}{\lambda_{\theta_{1}}} \tilde{\theta}_{1} \dot{\hat{\theta}}_{1}+\frac{1}{\lambda_{d_{1}}} \tilde{d}_{1} \dot{\hat{d}}_{1}+Z_{2} \dot{Z}_{2} .
\end{aligned}
$$

By appealing to the Nussbaum-gain technique to deal with the unknown control gains, the virtual control law and adaptive laws can be designed as:

$$
\begin{aligned}
& \alpha_{1}=N\left(\xi_{1}\right) \cdot \eta_{1}, \\
& \dot{\xi}_{1}=\vartheta_{s_{1}} \cdot \eta_{1} \\
& \eta_{1}=\frac{K_{1} \sin \left(\frac{\pi S_{1}^{2}}{k_{b_{1}}^{2}}\right)}{2 S_{1}}+\hat{\theta}_{1} \psi_{1}+l_{1} \vartheta_{s_{1}}+\hat{d}_{1} \tanh \left(\frac{\vartheta_{s_{1}}}{\varepsilon}\right)-\dot{y}_{d}, \\
& \dot{\hat{d}}_{1}=\lambda_{d_{1}} \vartheta_{s_{1}} \tanh \left(\frac{\vartheta_{s_{1}}}{\varepsilon}\right)-\sigma_{d_{1}} \hat{d}_{1}, \\
& \dot{\hat{\theta}}_{1}=\lambda_{\theta_{1}} \vartheta_{s_{1}} \psi_{1}-\sigma_{\theta_{1}} \hat{\theta}_{1},
\end{aligned}
$$


where $K_{1}, \sigma_{d_{1}}$ and $\sigma_{\theta_{1}}$ are positive constants.

Remark 6. Note that, by invoking L'Hospital rule, the first term of $\eta_{1}$ will tend to zero when $S_{1} \rightarrow 0$ :

$$
\lim _{S_{1} \rightarrow 0} \frac{K_{1} \sin \left(\frac{\pi S_{1}^{2}}{k_{b_{1}}^{2}}\right)}{2 S_{1}} \rightarrow 0 .
$$

Hence, the first term of $\eta_{1}$ will not be singular even if $S_{1}$ close to zero. In simulation, we should take it to be zero when $\left|S_{1}\right|<\epsilon$ for some small $\epsilon$, because " $\frac{0}{0}$ " cannot be computed in a digital computer.

Since $\alpha_{1}$ is continuous, differentiable and bounded, so the time derivative of $\alpha_{1}$ is bounded. By appealing to the equations (11) and (12), using the Young's inequality, the term of $Z_{2} \dot{Z}_{2}$ in (24) can be dealt with as follows

$$
\begin{aligned}
Z_{2} \dot{Z}_{2} & =Z_{2}\left(\dot{\alpha}_{2 f}-\dot{\alpha}_{1}\right)=Z_{2}\left(-\frac{Z_{2}}{\tau_{2}}-\dot{\alpha}_{1}\right) \\
& \leq-\frac{Z_{2}^{2}}{\tau_{2}}+\left|Z_{2}\right| m_{1} \\
& \leq\left(\frac{1}{4}-\frac{1}{\tau_{2}}\right) Z_{2}^{2}+m_{1}^{2}
\end{aligned}
$$

where $m_{1}$ is the supremum of $\left|\dot{\alpha}_{1}\right|$. Substituting (25)-(30) into (24), after some deformation, we have

$$
\begin{aligned}
\dot{V}_{1} \leq & \left(g_{1} N\left(\xi_{1}\right)+1\right) \dot{\xi}_{1}-K_{1} \tan \left(\frac{\pi S_{1}^{2}}{2 k_{b_{1}}^{2}}\right)+\frac{\bar{g}_{1}^{2}}{2 l_{1}} S_{2}^{2} \\
& +\left(\frac{1}{4}+\frac{\bar{g}_{1}^{2}}{2 l_{1}}-\frac{1}{\tau_{2}}\right) Z_{2}^{2}+m_{1}^{2}+d_{1} k_{\epsilon} \varepsilon-\frac{\sigma_{d_{1}}}{\lambda_{d_{1}}} \tilde{d}_{1} \hat{d}_{1}-\frac{\sigma_{\theta_{1}}}{\lambda_{\theta_{1}}} \tilde{\theta}_{1} \hat{\theta}_{1} .
\end{aligned}
$$

Using the Young's inequality, the terms $-\frac{\sigma_{d_{1}}}{\lambda_{d_{1}}} \tilde{d}_{1} \hat{d}_{1}$ and $-\frac{\sigma_{\theta_{1}}}{\lambda_{\theta_{1}}} \tilde{\theta}_{1} \hat{\theta}_{1}$ in (31) can be handled as:

$$
\begin{aligned}
-\frac{\sigma_{d_{1}}}{\lambda_{d_{1}}} \tilde{d}_{1} \hat{d}_{1} \leq-\frac{\sigma_{d_{1}}}{2 \lambda_{d_{1}}} \tilde{d}_{1}^{2}+\frac{\sigma_{d_{1}}}{2 \lambda_{d_{1}}} d_{1}^{2}, \\
-\frac{\sigma_{\theta_{1}}}{\lambda_{\theta_{1}}} \tilde{\theta}_{1} \hat{\theta}_{1} \leq-\frac{\sigma_{\theta_{1}}}{2 \lambda_{\theta_{1}}} \tilde{\theta}_{1}^{2}+\frac{\sigma_{\theta_{1}}}{2 \lambda_{\theta_{1}}} \theta_{1}^{2} .
\end{aligned}
$$

Substituting (32) and (33) into (31), it follows that

$$
\begin{aligned}
\dot{V}_{1} \leq & \left(g_{1} N\left(\xi_{1}\right)+1\right) \dot{\xi}_{1}-K_{1} \tan \left(\frac{\pi S_{1}^{2}}{2 k_{b_{1}}^{2}}\right) \\
& -\left(\frac{1}{\tau_{2}}-\frac{1}{4}-\frac{\bar{g}_{1}^{2}}{2 l_{1}}\right) Z_{2}^{2}+\frac{\bar{g}_{1}^{2}}{2 l_{1}} S_{2}^{2}-\frac{\sigma_{\theta_{1}}}{2 \lambda_{\theta_{1}}} \tilde{\theta}_{1}^{2}-\frac{\sigma_{d_{1}}}{2 \lambda_{d_{1}}} \tilde{d}_{1}^{2}+c_{1},
\end{aligned}
$$

where $c_{1}=m_{1}^{2}+d_{1} k_{\epsilon} \varepsilon+\frac{\sigma_{d_{1}}}{2 \lambda_{d_{1}}} d_{1}^{2}+\frac{\sigma_{\theta_{1}}}{2 \lambda_{\theta_{1}}} \theta_{1}^{2}$ is the clustered constant term. Let $\beta_{1}=\min \left\{\frac{\pi K_{1}}{k_{b_{1}}^{2}},\left(\frac{2}{\tau_{2}}-\frac{1}{2}-\right.\right.$ $\left.\left.\frac{\bar{g}_{1}^{2}}{l_{1}}\right), \sigma_{\theta_{1}}, \sigma_{d_{1}}\right\}$, notice the composition of $V_{1}$ in (19), one has

$$
\dot{V}_{1} \leq-\beta_{1} V_{1}+\left(g_{1} N\left(\xi_{1}\right)+1\right) \dot{\xi}_{1}+\frac{\bar{g}_{1}^{2}}{2 l_{1}} S_{2}^{2}+c_{1} .
$$


Multiplying both sides of (35) by $e^{\beta_{1} t}$ leads to

$$
\frac{d\left(V_{1}(t) e^{\beta_{1} t}\right)}{d t} \leq\left(g_{1} N\left(\xi_{1}\right)+1\right) \dot{\xi}_{1} e^{\beta_{1} t}+\frac{\bar{g}_{1}^{2}}{2 l_{1}} S_{2}^{2} e^{\beta_{1} t}+c_{1} e^{\beta_{1} t}
$$

Integrating the above inequality over $[0, t)$, it yields

$$
V_{1}(t) \leq e^{-\beta_{1} t} V_{1}(0)+e^{-\beta_{1} t} \int_{0}^{t}\left(g_{1} N\left(\xi_{1}\right)+1\right) \dot{\xi}_{1} e^{\beta_{1} \tau} d \tau+\int_{0}^{t} c_{1} e^{-\beta_{1}(t-\tau)} d \tau+\int_{0}^{t} \frac{\bar{g}_{1}^{2}}{2 l_{1}} S_{2}^{2} e^{-\beta_{1}(t-\tau)} d \tau(37)
$$

By invoking the Lemma 2, it can be concluded that $V_{1}(t), \xi_{1}(t)$ and $\int_{0}^{t} g_{1} N\left(\xi_{1}\right) \dot{\xi}_{1} d \tau$ are all bounded on $[0, t)$ as long as the last term of (37) is bounded. As a result, $V_{1}^{*}$ will be bounded and then $\left|S_{1}\right|<k_{b_{1}}$ holds. Therefore, the problem boils down to the boundedness of $S_{2}$, which will be obtained in Step 2.

Step $j(j=2, \cdots, n-1)$. From Step 2 onwards, a similar design procedure can be involved recursively at each step. Considering the $x_{j}$-subsystem of system (6), take the time derivative of $S_{j}$ based on equations (6), (9), (11) and (12), it yields

$$
\begin{aligned}
\dot{S}_{j} & =\dot{x}_{j}-\dot{\alpha}_{j f} \\
& =\theta_{j} \psi_{j}+g_{j}\left(S_{j+1}+Z_{j+1}+\alpha_{j}\right)+D_{j}+\frac{Z_{j}}{\tau_{j}} .
\end{aligned}
$$

Analogous to the design approach in Step 1, the following Lyapunov function candidate is employed:

$$
V_{j}=V_{j}^{*}+\frac{1}{2 \lambda_{\theta_{j}}} \tilde{\theta}_{j}^{2}+\frac{1}{2 \lambda_{d_{j}}} \tilde{d}_{j}^{2}+\frac{1}{2} Z_{j+1}^{2}
$$

where $\lambda_{\theta_{j}}$ and $\lambda_{d_{j}}$ are positive constants. According to (16) and (38), the time derivative of $V_{j}^{*}$ can be written as

$$
\dot{V}_{j}^{*}=\vartheta_{s_{j}}\left(\theta_{j} \psi_{j}+g_{j}\left(S_{j+1}+Z_{j+1}+\alpha_{j}\right)+D_{j}+\frac{Z_{j}}{\tau_{j}}\right) .
$$

By invoking Assumption 1 and Lemma 1, it follows that

$$
\vartheta_{s_{j}} D_{j} \leq\left|\vartheta_{s_{j}}\right| d_{j} \leq d_{j}\left(k_{\epsilon} \varepsilon+\vartheta_{s_{j}} \tanh \left(\frac{\vartheta_{s_{j}}}{\varepsilon}\right)\right) .
$$

Utilizing the Young's inequality, it leads to

$$
\begin{aligned}
& g_{j} \vartheta_{s_{j}} S_{j+1} \leq \frac{l_{j}}{2} \vartheta_{s_{j}}^{2}+\frac{\bar{g}_{j}^{2}}{2 l_{j}} S_{j+1}^{2}, \\
& g_{j} \vartheta_{s_{j}} Z_{j+1} \leq \frac{l_{j}}{2} \vartheta_{s_{j}}^{2}+\frac{\bar{g}_{j}^{2}}{2 l_{j}} Z_{j+1}^{2},
\end{aligned}
$$

where $l_{j}$ is a positive constant. With the help of (39)-(43), differentiating $V_{j}$ with respect to time yields

$$
\begin{aligned}
\dot{V}_{j} \leq & \vartheta_{s_{j}} \cdot\left(\theta_{j} \psi_{j}+g_{j} \alpha_{j}+\frac{Z_{j}}{\tau_{j}}\right)+l_{j} \vartheta_{s_{j}}^{2}+\frac{\bar{g}_{j}^{2}}{2 l_{j}} S_{j+1}^{2}+\frac{\bar{g}_{j}^{2}}{2 l_{j}} Z_{j+1}^{2} \\
& +d_{j}\left(k_{\epsilon} \varepsilon+\vartheta_{s_{j}} \tanh \left(\frac{\vartheta_{s_{j}}}{\varepsilon}\right)\right)+\frac{1}{\lambda_{\theta_{j}}} \tilde{\theta}_{j} \dot{\hat{\theta}}_{j}+\frac{1}{\lambda_{d_{j}}} \tilde{d}_{j} \dot{\hat{d}}_{j}+Z_{j+1} \dot{Z}_{j+1} .
\end{aligned}
$$


By employing the Nussbaum-gain technique to handle the unknown control gain, the virtual control law and adaptive laws are designed as:

$$
\begin{aligned}
& \alpha_{j}=N\left(\xi_{j}\right) \cdot \eta_{j}, \\
& \dot{\xi}_{j}=\vartheta_{s_{j}} \cdot \eta_{j}, \\
& \eta_{j}=\frac{K_{j} \sin \left(\frac{\pi S_{j}^{2}}{k_{b_{j}}^{2}}\right)}{2 S_{j}}+\hat{\theta}_{j} \psi_{j}+l_{j} \vartheta_{s_{j}}+\hat{d}_{j} \tanh \left(\frac{\vartheta_{s_{j}}}{\varepsilon}\right)+\frac{Z_{j}}{\tau_{j}}, \\
& \dot{\hat{d}}_{j}=\lambda_{d_{j}} \vartheta_{s_{j}} \tanh \left(\frac{\vartheta_{s_{j}}}{\varepsilon}\right)-\sigma_{d_{j}} \hat{d}_{j}, \\
& \dot{\hat{\theta}}_{j}=\lambda_{\theta_{j}} \vartheta_{s_{j}} \psi_{j}-\sigma_{\theta_{j}} \hat{\theta}_{j},
\end{aligned}
$$

where $K_{j}, \sigma_{d_{j}}$ and $\sigma_{\theta_{j}}$ are positive parameters. Analogous to the discussion in Step 1, the boundedness of $\dot{\alpha}_{j}$ also can be verified. According to the Young's inequality, with the help of the equations (11) and (12), $Z_{j+1} \dot{Z}_{j+1}$ in (44) can be dealt with as follows

$$
\begin{aligned}
Z_{j+1} \dot{Z}_{j+1} & =Z_{j+1}\left(\dot{\alpha}_{(j+1) f}-\dot{\alpha}_{j}\right)=Z_{j+1}\left(-\frac{Z_{j+1}}{\tau_{j+1}}-\dot{\alpha}_{j}\right) \\
& \leq-\frac{Z_{j+1}^{2}}{\tau_{j+1}}+\left|Z_{j+1}\right| m_{j} \leq\left(\frac{1}{4}-\frac{1}{\tau_{j+1}}\right) Z_{j+1}^{2}+m_{j}^{2},
\end{aligned}
$$

where $m_{j}$ is a positive constant. Substituting (45)-(50) into (44), adding and subtracting $\dot{\xi}_{j}$ on the right side of (44), after some deformation, one has

$$
\begin{aligned}
\dot{V}_{j} \leq & \left(g_{j} N\left(\xi_{j}\right)+1\right) \dot{\xi}_{j}-K_{j} \tan \left(\frac{\pi S_{j}^{2}}{2 k_{b_{j}}^{2}}\right)+\frac{\bar{g}_{j}^{2}}{2 l_{j}} S_{j+1}^{2} \\
& -\left(\frac{1}{\tau_{j+1}}-\frac{1}{4}-\frac{\bar{g}_{j}^{2}}{2 l_{j}}\right) Z_{j+1}^{2}+m_{j}^{2}+d_{j} k_{\epsilon} \varepsilon-\frac{\sigma_{d_{j}}}{\lambda_{d_{j}}} \tilde{d}_{j} \hat{d}_{j}-\frac{\sigma_{\theta_{j}}}{\lambda_{\theta_{j}}} \tilde{\theta}_{j} \hat{\theta}_{j} .
\end{aligned}
$$

Using the Young's inequality, the terms $-\frac{\sigma_{d_{j}}}{\lambda_{d_{j}}} \tilde{d}_{j} \hat{d}_{j}$ and $-\frac{\sigma_{\theta_{j}}}{\lambda_{\theta_{j}}} \tilde{\theta}_{j} \hat{\theta}_{j}$ in (51) can be handled as:

$$
\begin{aligned}
& -\frac{\sigma_{d_{j}}}{\lambda_{d_{j}}} \tilde{d}_{j} \hat{d}_{j} \leq-\frac{\sigma_{d_{j}}}{2 \lambda_{d_{j}}} \tilde{d}_{j}^{2}+\frac{\sigma_{d_{j}}}{2 \lambda_{d_{j}}} d_{j}^{2}, \\
& -\frac{\sigma_{\theta_{j}}}{\lambda_{\theta_{j}}} \tilde{\theta}_{j} \hat{\theta}_{j} \leq-\frac{\sigma_{\theta_{j}}}{2 \lambda_{\theta_{j}}} \tilde{\theta}_{j}^{2}+\frac{\sigma_{\theta_{j}}}{2 \lambda_{\theta_{j}}} \theta_{j}^{2} .
\end{aligned}
$$

Substituting (52) and (53) into (51), it follows that

$$
\begin{aligned}
\dot{V}_{j} \leq & \left(g_{j} N\left(\xi_{j}\right)+1\right) \dot{\xi}_{j}-K_{j} \tan \left(\frac{\pi S_{j}^{2}}{2 k_{b_{j}}^{2}}\right)-\left(\frac{1}{\tau_{j+1}}-\frac{1}{4}-\frac{\bar{g}_{j}^{2}}{2 l_{j}}\right) Z_{j+1}^{2} \\
& +\frac{\bar{g}_{j}^{2}}{2 l_{j}} S_{j+1}^{2}-\frac{\sigma_{\theta_{j}}}{2 \lambda_{\theta_{j}}} \tilde{\theta}_{j}^{2}-\frac{\sigma_{d_{j}}}{2 \lambda_{d_{j}}} \tilde{d}_{j}^{2}+c_{j},
\end{aligned}
$$

where $c_{j}=m_{j}^{2}+d_{j} k_{\epsilon} \varepsilon+\frac{\sigma_{d_{j}}}{2 \lambda_{d_{j}}} d_{j}^{2}+\frac{\sigma_{\theta_{j}}}{2 \lambda_{\theta_{j}}} \theta_{j}^{2}$ is the clustered constant term. Given $\beta_{j}=\min \left\{\frac{\pi K_{j}}{k_{b_{j}}^{2}},\left(\frac{2}{\tau_{j+1}}-\right.\right.$ $\left.\left.\frac{1}{2}-\frac{\bar{g}_{j}^{2}}{l_{j}}\right), \sigma_{\theta_{j}}, \sigma_{d_{j}}\right\}$, consider the composition of $V_{j}$ in (39), one has

$$
\dot{V}_{j} \leq-\beta_{j} V_{j}+\left(g_{j} N\left(\xi_{j}\right)+1\right) \dot{\xi}_{j}+\frac{\bar{g}_{j}^{2}}{2 l_{j}} S_{j+1}^{2}+c_{j}
$$


Multiplying both sides of inequality (55) by $e^{\beta_{j} t}$, and then integrating both sides over $[0, t)$, it yields

$$
\left.V_{j}(t) \leq e^{-\beta_{j} t} V_{j}(0)+e^{-\beta_{j} t} \int_{0}^{t}\left(g_{j} N\left(\xi_{j}\right)+1\right) \dot{\xi}_{j} e^{\beta_{j} \tau} d \tau+\int_{0}^{t} c_{j} e^{-\beta_{j}(t-\tau)} d \tau+\int_{0}^{t} \frac{\bar{g}_{j}^{2}}{2 l_{j}} S_{j+1}^{2} e^{-\beta_{j}(t-\tau)} d \star 56\right)
$$

Invoking the Lemma 2, it can be seen from the above result, $V_{j}(t), \xi_{j}(t)$ and $\int_{0}^{t} g_{j} N\left(\xi_{j}\right) \dot{\xi}_{j} d \tau$ are all bounded on $[0, t)$ as long as the last term of (56) is bounded. As a result, $V_{j}^{*}$ is bounded and then $\left|S_{j}\right|<k_{b_{j}}$ holds. Therefore, the problem boils down to the boundedness of $S_{j+1}$, which will be obtained in Step $j+1$.

Step $n$. Considering the $x_{n}$-subsystem of system (6), take the time derivative of $S_{n}$ based on equations (6) and (9)-(12), it yields

$$
\begin{aligned}
\dot{S}_{n} & =\dot{x}_{n}-\dot{\alpha}_{n f} \\
& =\theta_{n} \psi_{n}+g_{n} \hbar(v)+\bar{D}_{n}+\frac{Z_{n}}{\tau_{n}} \\
& =\theta_{n} \psi_{n}+g_{n}\left(S_{n+1}+Z_{n+1}+\alpha_{n}\right)+\bar{D}_{n}+\frac{Z_{n}}{\tau_{n}} .
\end{aligned}
$$

For the external disturbance term $\bar{D}_{n}$, invoking Assumption 1-2 and equation (5), one has

$$
\left|\bar{D}_{n}\right|=\left|D_{n}+g_{n} \rho(v)\right| \leq d_{n}+\bar{g}_{n} \bar{\rho}=\bar{d}_{n}
$$

where $\bar{d}_{n}$ is a positive constant. Choose the Lyapunov function candidate $V_{n}$ as:

$$
V_{n}=V_{n}^{*}+\frac{1}{2 \lambda_{\theta_{n}}} \tilde{\theta}_{n}^{2}+\frac{1}{2 \lambda_{d_{n}}} \tilde{\tilde{d}}_{n}^{2}+\frac{1}{2} Z_{n+1}^{2}
$$

where $\lambda_{\theta_{n}}$ and $\lambda_{d_{n}}$ are positive constants, $\tilde{\bar{d}}_{n}=\hat{\bar{d}}_{n}-\bar{d}_{n}$ with $\hat{\bar{d}}_{n}$ be an estimate of $\bar{d}_{n}$. According to (16) and (57), taking the time derivative of $V_{n}^{*}$, it yields

$$
\dot{V}_{n}^{*}=\vartheta_{s_{n}}\left(\theta_{n} \psi_{n}+g_{n}\left(S_{n+1}+Z_{n+1}+\alpha_{n}\right)+\bar{D}_{n}+\frac{Z_{n}}{\tau_{n}}\right) .
$$

By invoking Assumption 1 and Lemma 1, it follows that

$$
\vartheta_{s_{n}} \bar{D}_{n} \leq\left|\vartheta_{s_{n}}\right| \bar{d}_{n} \leq \bar{d}_{n}\left(k_{\epsilon} \varepsilon+\vartheta_{s_{n}} \tanh \left(\frac{\vartheta_{s_{n}}}{\varepsilon}\right)\right) .
$$

Utilizing the Young's inequality, we can obtain:

$$
\begin{aligned}
& g_{n} \vartheta_{s_{n}} S_{n+1} \leq \frac{l_{n}}{2} \vartheta_{s_{n}}^{2}+\frac{\bar{g}_{n}^{2}}{2 l_{n}} S_{n+1}^{2}, \\
& g_{n} \vartheta_{s_{n}} Z_{n+1} \leq \frac{l_{n}}{2} \vartheta_{s_{n}}^{2}+\frac{\bar{g}_{n}^{2}}{2 l_{n}} Z_{n+1}^{2},
\end{aligned}
$$

where $l_{n}$ is a positive parameter. With the help of (59)-(63), taking the time derivative of $V_{n}$, it follows that

$$
\begin{aligned}
\dot{V}_{n} \leq & \vartheta_{s_{n}} \cdot\left(\theta_{n} \psi_{n}+g_{n} \alpha_{n}+\frac{Z_{n}}{\tau_{n}}\right)+l_{n} \vartheta_{s_{n}}^{2}+\frac{\bar{g}_{n}^{2}}{2 l_{n}} Z_{n+1}^{2}+\bar{d}_{n}\left(k_{\epsilon} \varepsilon+\vartheta_{s_{n}} \tanh \left(\frac{\vartheta_{s_{n}}}{\varepsilon}\right)\right) \\
& +\frac{\bar{g}_{n}^{2}}{2 l_{n}} S_{n+1}^{2}+\frac{1}{\lambda_{\theta_{n}}} \tilde{\theta}_{n} \dot{\hat{\theta}}_{n}+\frac{1}{\lambda_{d_{n}}} \tilde{\bar{d}}_{n} \dot{\overline{\bar{d}}}_{n}+Z_{n+1} \dot{Z}_{n+1} .
\end{aligned}
$$


In the same way as before, the following Nussbaum type controller and adaptive laws are designed

$$
\begin{aligned}
\alpha_{n} & =N\left(\xi_{n}\right) \cdot \eta_{n}, \\
\dot{\xi}_{n} & =\vartheta_{s_{n}} \cdot \eta_{n}, \\
\eta_{n} & =\frac{K_{n} \sin \left(\frac{\pi S_{n}^{2}}{k_{b_{n}}^{2}}\right)}{2 S_{n}}+\hat{\theta}_{n} \psi_{n}+l_{n} \vartheta_{s_{n}}+\hat{\bar{d}}_{n} \tanh \left(\frac{\vartheta_{s_{n}}}{\varepsilon}\right)+\frac{Z_{n}}{\tau_{n}}, \\
\dot{\bar{d}}_{n} & =\lambda_{d_{n}} \vartheta_{s_{n}} \tanh \left(\frac{\vartheta_{s_{n}}}{\varepsilon}\right)-\sigma_{d_{n}} \hat{\bar{d}}_{n}, \\
\dot{\hat{\theta}}_{n} & =\lambda_{\theta_{n}} \vartheta_{s_{n}} \psi_{n}-\sigma_{\theta_{n}} \hat{\theta}_{n},
\end{aligned}
$$

where $K_{n}, \sigma_{d_{n}}$ and $\sigma_{\theta_{n}}$ are positive parameters. In the same way as the previous steps, the boundedness of $\dot{\alpha}_{n}$ can be verified. By appealing to the equations (11) and (12), the term of $Z_{n+1} \dot{Z}_{n+1}$ in (64) can be dealt with as follows by the Young's inequality,

$$
\begin{aligned}
Z_{n+1} \dot{Z}_{n+1} & =Z_{n+1}\left(\dot{\alpha}_{(n+1) f}-\dot{\alpha}_{n}\right)=Z_{n+1}\left(-\frac{Z_{n+1}}{\tau_{n+1}}-\dot{\alpha}_{n}\right) \\
& \leq-\frac{Z_{n+1}^{2}}{\tau_{n+1}}+\left|Z_{n+1}\right| m_{n} \leq\left(\frac{1}{4}-\frac{1}{\tau_{n+1}}\right) Z_{n+1}^{2}+m_{n}^{2}
\end{aligned}
$$

where $m_{n}$ is a positive constant. Substituting (65)-(70) into (64), adding and subtracting the term $\dot{\xi}_{n}$ on the right side of (64), we have

$$
\begin{aligned}
\dot{V}_{n} \leq & \left(g_{n} N\left(\xi_{n}\right)+1\right) \dot{\xi}_{n}-K_{n} \tan \left(\frac{\pi S_{n}^{2}}{2 k_{b_{n}}^{2}}\right)+\frac{\bar{g}_{n}^{2}}{2 l_{n}} S_{n+1}^{2}-\left(\frac{1}{\tau_{n+1}}-\frac{1}{4}-\frac{\bar{g}_{n}^{2}}{2 l_{n}}\right) Z_{n+1}^{2} \\
& +m_{n}^{2}+\bar{d}_{n} k_{\epsilon} \varepsilon-\frac{\sigma_{d_{n}}}{\lambda_{d_{n}}} \tilde{\bar{d}}_{n} \hat{\bar{d}}_{n}-\frac{\sigma_{\theta_{n}}}{\lambda_{\theta_{n}}} \tilde{\theta}_{n} \hat{\theta}_{n} .
\end{aligned}
$$

Using the Young's inequality, the terms $-\frac{\sigma_{d_{n}}}{\lambda_{d_{n}}} \tilde{\bar{d}}_{n} \hat{d}_{n}$ and $-\frac{\sigma_{\theta_{n}}}{\lambda_{\theta_{n}}} \tilde{\theta}_{n} \hat{\theta}_{n}$ in (71) can be handled as:

$$
\begin{aligned}
& -\frac{\sigma_{d_{n}}}{\lambda_{d_{n}}} \tilde{\bar{d}}_{n} \hat{\bar{d}}_{n} \leq-\frac{\sigma_{d_{n}}}{2 \lambda_{d_{n}}} \tilde{\bar{d}}_{n}^{2}+\frac{\sigma_{d_{n}}}{2 \lambda_{d_{n}}} \bar{d}_{n}^{2} \\
& -\frac{\sigma_{\theta_{n}}}{\lambda_{\theta_{n}}} \tilde{\theta}_{n} \hat{\theta}_{n} \leq-\frac{\sigma_{\theta_{n}}}{2 \lambda_{\theta_{n}}} \tilde{\theta}_{n}^{2}+\frac{\sigma_{\theta_{n}}}{2 \lambda_{\theta_{n}}} \theta_{n}^{2} .
\end{aligned}
$$

Substituting (72) and (73) into (71), it follows that

$$
\dot{V}_{n} \leq-\beta_{n} V_{n}+\left(g_{n} N\left(\xi_{n}\right)+1\right) \dot{\xi}_{n}+\frac{\bar{g}_{n}^{2}}{2 l_{n}} S_{n+1}^{2}+c_{n}
$$

where $c_{n}=m_{n}^{2}+\bar{d}_{n} k_{\epsilon} \varepsilon+\frac{\sigma_{d_{n}}}{2 \lambda_{d_{n}}} \bar{d}_{n}^{2}+\frac{\sigma_{\theta_{n}}}{2 \lambda_{\theta_{n}}} \theta_{n}^{2}$ is the constant part, and $\beta_{n}=\min \left\{\frac{\pi K_{n}}{k_{b_{n}}^{2}},\left(\frac{2}{\tau_{n+1}}-\frac{1}{2}-\right.\right.$ $\left.\left.\frac{\bar{g}_{n}^{2}}{l_{n}}\right), \sigma_{\theta_{n}}, \sigma_{d_{n}}\right\}$. Multiplying both sides of (74) by $e^{\beta_{n} t}$, then integrating it over $[0, t)$, it yields

$$
\begin{aligned}
V_{n}(t) & \leq e^{-\beta_{n} t} V_{n}(0)+\int_{0}^{t} c_{n} e^{-\beta_{n}(t-\tau)} d \tau+e^{-\beta_{n} t} \int_{0}^{t}\left(g_{n} N\left(\xi_{n}\right)+1\right) \dot{\xi}_{n} e^{\beta_{n} \tau} d \tau \\
& +\int_{0}^{t} \frac{\bar{g}_{n}^{2}}{2 l_{n}} S_{n+1}^{2} e^{-\beta_{n}(t-\tau)} d \tau
\end{aligned}
$$


It can be seen from the above result, invoking the Lemma 2, as long as the last term of (75) is bounded, then $V_{n}(t), \xi_{n}(t)$ and $\int_{0}^{t} g_{n} N\left(\xi_{n}\right) \dot{\xi}_{n} d \tau$ are all bounded on $[0, t)$. As a result, $V_{n}^{*}$ is bounded and then $\left|S_{n}\right|<k_{b_{n}}$ holds. Therefore, the problem boils down to the boundedness of $S_{n+1}$, which will be obtained in Step $n+1$.

Step $n+1$. Considering the auxiliary $(n+1)$-subsystem of system (6). Since the virtual error $S_{n+1}$ only needs to be bounded, rather than restricted to a predefined region, the BLF is no longer required in this step. Define the following quadratic Lyapunov function candidate:

$$
V_{n+1}=\frac{1}{2} S_{n+1}^{2}
$$

From equations (3), (10) and (12), computing the time derivative of $V_{n+1}$, it yields

$$
\dot{V}_{n+1}=S_{n+1}\left(G(t) \dot{v}-\dot{\alpha}_{(n+1) f}\right)=S_{n+1}\left(G(t)(\mu-\zeta v)+\frac{Z_{n+1}}{\tau_{n+1}}\right),
$$

where $G(t)=\frac{\partial \hbar(v)}{\partial v}=\frac{4}{\left(e^{\frac{v}{u}}+e^{-\frac{v}{u}}\right)^{2}}$. Since the control coefficient is still unknown, the auxiliary control law can be designed as

$$
\begin{aligned}
\mu & =N\left(\xi_{n+1}\right) \cdot \eta_{n+1} \\
\dot{\xi}_{n+1} & =S_{n+1} \cdot \eta_{n+1} \\
\eta_{n+1} & =K_{n+1} S_{n+1}-G(t) \zeta v+\frac{Z_{n+1}}{\tau_{n+1}}
\end{aligned}
$$

where $K_{n+1}$ is a positive parameter. Substituting (78)-(80) into (77), adding and subtracting the term $\dot{\xi}_{n+1}$ on the right side of (77), one has

$$
\begin{aligned}
\dot{V}_{n+1} & =-K_{n+1} S_{n+1}^{2}+\left(G(t) N\left(\xi_{n+1}\right)+1\right) \dot{\xi}_{n+1} \\
& \leq-\beta_{n+1} V_{n+1}+\left(G(t) N\left(\xi_{n+1}\right)+1\right) \dot{\xi}_{n+1}
\end{aligned}
$$

where $\beta_{n+1}$ is a positive constant and supposing $\beta_{n+1} \leq 2 K_{n+1}$. Multiplying both sides of (81) by $e^{\beta_{n+1} t}$ and integrating it over $[0, t)$, it follows that

$$
V_{n+1}(t) \leq e^{-\beta_{n+1} t} V_{n+1}(0)+e^{-\beta_{n+1} t} \int_{0}^{t}\left(G(t) N\left(\xi_{n+1}\right)+1\right) \dot{\xi}_{n+1} e^{\beta_{n+1} \tau} d \tau
$$

By invoking the Lemma 2, because inequality (82) holds, then $V_{n+1}(t), \xi_{n+1}(t)$ and $\int_{0}^{t} G(t) N\left(\xi_{n+1}\right) \dot{\xi}_{n} d \tau$ are all bounded on $[0, t)$. As a result, $S_{n+1}$ is bounded, and then it is concluded that $V_{n}(t), \xi_{n}(t)$ and $\int_{0}^{t} g_{n} N\left(\xi_{n}\right) \dot{\xi}_{n} d \tau$ are all bounded on $[0, t)$ from Step $n$. Back to the previous steps one by one, we can summarize $V_{i}(t), \xi_{i}(t)$ and $\int_{0}^{t} g_{i} N\left(\xi_{i}\right) \dot{\xi}_{i} d \tau$ are all bounded on $\left[0, t_{f}\right)$, and $\left|S_{i}\right|<k_{b_{i}}(i=1, \cdots, n)$ hold. Furthermore, based on Remark $2, t$ can be extended to infinity.

\subsection{Theoretical results and proof}

Based on the adaptive controller design of the system (1) given in subsection $\mathrm{B}$, the following theorem is obtained, which summarizes the main results of this paper.

Theorem 1: Considering the state and input constrained uncertain nonlinear system (1) with unknown control coefficients under Assumptions 1-3. Supposing that the input saturation signal is constrained by $\bar{u}$, 
and the initial states satisfy $x_{j}(0) \in \Omega_{x_{j}}(j=1, \cdots, n)$. By constructing the expanded system (6), the virtual/actual control laws and adaptive laws shown in (45)-(49), (65)-(69) and (78)-(80) are designed, which make the following properties hold.

(i). All the signals of the closed-loop system are bounded;

(ii). All the states are always within the predefined constrained regions;

(iii). The actual/virtual tracking errors $S_{j}(t)(j=1, \cdots, n)$ converge to a small neighbourhood of zero.

Proof: (i). From the adaptive controller design process given in subsection B , we know that $V_{j}(j=$ $1, \cdots, n)$ are bounded. Note that $V_{j}=\frac{k_{b_{j}}^{2}}{\pi} \tan \left(\frac{\pi S_{j}^{2}}{2 k_{b_{j}}^{2}}\right)+\frac{1}{2 \lambda_{\theta_{j}}} \tilde{\theta}_{j}^{2}+\frac{1}{2 \lambda_{d_{j}}} \tilde{d}_{j}^{2}+\frac{1}{2} Z_{j+1}^{2}$, so $S_{j}, \tilde{\theta}_{j}, \tilde{d}_{j}$ and $Z_{j+1}$ are all bounded. The boundedness of $\theta_{j}, \tilde{\theta}_{j}, d_{j}$, and $\tilde{d}_{j}$ ensure the boundedness of $\hat{\theta}_{j}$ and $\hat{d}_{j}$, and further make $\eta_{j}$ bounded. Since $\alpha_{j}=N\left(\xi_{j}\right) \eta_{j}$, the boundedness of $\xi_{j}$ and $\eta_{j}$ guarantee the boundedness of $\alpha_{j}$. As a result, $\alpha_{j f}$ is bounded. In the same way, $\mu$ is bounded. Then, $x_{j}(j=1, \cdots, n)$ are bounded. Conclusively, all the signals of the closed-loop system are bounded.

(ii). The boundedness of $y_{d}, \alpha_{j f}$ and $S_{j}$ have been stressed in (i). In view of $S_{1}=x_{1}-y_{d},\left|S_{1}\right| \leq k_{b_{1}}$ and $\left|y_{d}\right| \leq Y_{0}$, we can obtain $\left|x_{1}\right| \leq\left|S_{1}\right|+\left|y_{d}\right|<k_{b_{1}}+Y_{0}$. So, only given $k_{b_{1}}=k_{c_{1}}-Y_{0}$, then $\left|x_{1}\right|<k_{c_{1}}$ holds. Since $\alpha_{j f}$ is bounded, we assume there exist a positive constant $\bar{\alpha}_{j 0}$ which make $\left|\alpha_{j f}\right| \leq \bar{\alpha}_{j 0}$. In view of $S_{j}=x_{j}-\alpha_{j f},\left|S_{j}\right| \leq k_{b_{j}}$ and $\left|\alpha_{j f}\right| \leq \bar{\alpha}_{j 0}$, it follows that $\left|x_{j}\right| \leq\left|S_{j}\right|+\left|\alpha_{j f}\right|<k_{b_{j}}+\bar{\alpha}_{j 0}$. So, only given $k_{b_{j}}=k_{c_{j}}-\bar{\alpha}_{j 0}$, then $\left|x_{j}\right|<k_{c_{j}}(j=2, \cdots, n)$ hold. Thus we conclude that all the states can remain within the predefined constrained regions by proper selection of $k_{b_{j}}$.

(iii). According to (35) and the above analysis, we know

$$
\dot{V}_{1} \leq-\beta_{1} V_{1}+M_{1}
$$

where $M_{1}=\sup \left\{\left(g_{1} N\left(\xi_{1}\right)+1\right) \dot{\xi}_{1}+\frac{\bar{g}_{1}^{2}}{2 l_{1}} S_{2}^{2}+c_{1}\right\}$ is a positive constant. Integrating the both sides of (83) over $[0, t)$, it yields

$$
0 \leq V_{1} \leq \frac{M_{1}}{\beta_{1}}+\left(V_{1}(0)-\frac{M_{1}}{\beta_{1}}\right) e^{-\beta_{1} t}
$$

Since $V_{1}=\frac{k_{b_{1}}^{2}}{\pi} \tan \left(\frac{\pi S_{1}^{2}}{2 k_{b_{1}}^{2}}\right)+\frac{1}{2 \lambda_{\theta_{1}}} \tilde{\theta}_{1}^{2}+\frac{1}{2 \lambda_{d_{1}}} \tilde{d}_{1}^{2}+\frac{1}{2} Z_{2}^{2}$, it follows that

$$
\left|S_{1}\right| \leq \sqrt{\frac{2 M_{1}}{\beta_{1}}+2\left(V_{1}(0)-\frac{M_{1}}{\beta_{1}}\right) e^{-\beta_{1} t}} .
$$

Therefore, there exist a time constant $T>0$, for any $t>T$, such that $\left|S_{1}\right|=\left|x_{1}-y_{d}\right| \leq \omega_{1}$ holds with $\omega_{1}>$ $\sqrt{\frac{2 M_{1}}{\beta_{1}}}$. It indicates that the tracking error settles within the compact set $\Omega=\left\{\left|x_{1}-y_{d}\right| \leq \omega_{1}, \omega_{1}>\sqrt{\frac{2 M_{1}}{\beta_{1}}}\right\}$. The quantity $\sqrt{\frac{2 M_{1}}{\beta_{1}}}$ can be adjusted to be small through artfully adjusting the design parameters.

Similarly, from (55) and (74), it obtains

$$
\dot{V}_{j} \leq-\beta_{j} V_{j}+M_{j},(j=2, \cdots, n)
$$

where $M_{j}$ are positive constants. Then, integrating it over $[0, t]$, which leads to the following result

$$
\left|S_{j}\right| \leq \sqrt{\frac{2 M_{j}}{\beta_{j}}+2\left(V_{j}(0)-\frac{M_{j}}{\beta_{j}}\right) e^{-\beta_{j} t}} .
$$


Hence, there exist time constants $T_{j}>0$, for any $t>T_{j}$, such that $\left|S_{j}\right| \leq \omega_{j}$ hold with $\omega_{j}>\sqrt{\frac{2 M_{j}}{\beta_{j}}}$. Therefore, it is concluded that the actual/virtual tracking errors $S_{j}(j=1, n)$ converge to a small neighbourhood of zero.

\section{Simulation}

In this section, in order to demonstrate the effectiveness of the proposed control scheme, the following states and input constrained nonlinear system is considered:

$$
\left\{\begin{array}{l}
\dot{x}_{1}=\theta_{1} \psi_{1}\left(\bar{x}_{1}\right)+g_{1}\left(\bar{x}_{1}\right) x_{2}+D_{1}(x, t) \\
\dot{x}_{2}=\theta_{2} \psi_{2}\left(\bar{x}_{2}\right)+g_{2}\left(\bar{x}_{2}\right) u(v)+D_{2}(x, t) \\
y=x_{1}
\end{array}\right.
$$

where $\psi_{1}\left(x_{1}\right)=x_{1}^{2}, \psi_{2}\left(\bar{x}_{2}\right)=0.2 x_{1} x_{2}+x_{1}, \theta_{1}$ and $\theta_{2}$ are parametric uncertainties, $D_{1}$ and $D_{2}$ are external disturbances.

The control objective is to design a saturation controller with constraint $\bar{u}=0.5$, which make the output $y(t)$ to track the given reference signal $y_{d}(t)=0.5 * \cos (t / 2)+0.2 * \sin (t)$ as closely as possible, and the states within the constrained regions: $\Omega_{x}=\left\{\left|x_{1}\right|<k_{c_{1}}=0.8,\left|x_{2}\right|<k_{c_{2}}=2\right\}$ for all the system running time.

Remark 7. In order to fully verify the effectiveness of our control scheme for system with unknown control direction and unknown disturbance, simulations will be carried out under three cases. Thus to show that our controller is effective for arbitrary control direction and external disturbance. For comparing the control effect better, the initial values and parameters selection are the same in all three cases. Specifically, the initial values are given as $x_{1}(0)=0.6, x_{2}(0)=-0.1, \theta_{1}(0)=0.1, \theta_{2}(0)=0.2, \zeta_{1}(0)=0.3, \zeta_{2}(0)=0.5$. Additionally, the design parameters are selected as $K_{1}=K_{2}=15, l_{1}=1, \tau_{2}=0.2, \lambda_{\theta_{1}}=\lambda_{\theta_{2}}=1$, $\sigma_{\theta_{1}}=\sigma_{\theta_{2}}=0.01, \varepsilon=0.01$.

Case one: $g_{1}=-1-\sin \left(x_{1}\right), g_{2}=-1.5-\sin \left(x_{1} x_{2}\right) ; D_{1}=D_{2}=0$.

This is the normal case without external disturbance, the direction of the gain function $g_{1}$ and $g_{2}$ are the same. Using the matlab routine we get the constraints of the virtual errors are $k_{b_{1}}=k_{c_{1}}-\sup \left\{\left|y_{d}\right|\right\}=0.4$ and $k_{b_{2}}=1.73$. In light of the design procedure proposed in section 2.2, the state feedback controller can be constructed and the simulation results are shown in Fig. 1-7. Fig. 1 shows the trajectory of the system output following the reference signal. On the one hand, it illustrates the accuracy of tracking performance, on the other hand, it also shows that the system state $x_{1}$ is always within the constrained region. The trajectory of the another state $x_{2}$ is shown in Fig. 2, it also within its predefined constrained region for all the time. Fig. 3 gives the trajectories of the uncertain parameters. Fig. 4 illustrates the trajectories of the Nussbaum functions and their arguments. The system input signal is given in Fig. 5 and Fig. 6, of which Fig. 6 is the input saturation signal. It can be seen that the input of the system is always between -0.5 and 0.5 , so as to meet the requirements of saturation. The trajectories of the tracking errors are shown in Fig. 7. It indicates that the output tracking error converges to a very small neighbourhood of zero. Then, the state feedback controller can be constructed as

$$
\alpha_{1}=N\left(\xi_{1}\right) \cdot \eta_{1}
$$



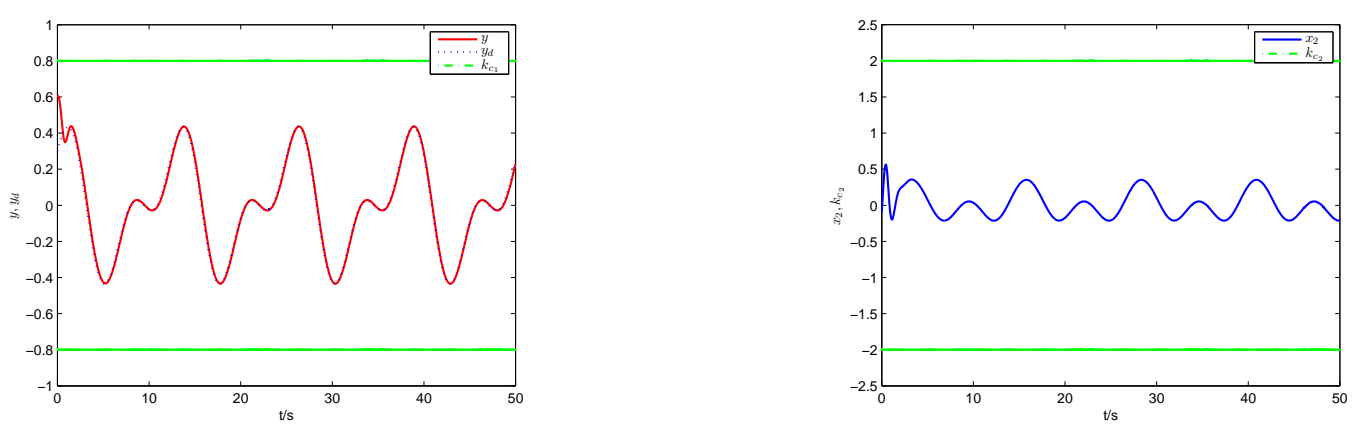

Figure 1: Trajectories of $y, y_{d}$ and their constraint(case Figure 2: Trajectorie of $x_{2}$ and its constraint(case one). one)

$$
\begin{gathered}
\dot{\xi}_{1}=\vartheta_{s_{1}} \cdot \eta_{1} \\
\eta_{1}=\frac{K_{1} \sin \left(\frac{\pi S_{1}^{2}}{k_{b_{1}}^{2}}\right)}{2 S_{1}}+\hat{\theta}_{1} \psi_{1}+l_{1} \vartheta_{s_{1}}-\dot{y}_{d} \\
\dot{\hat{\theta}}_{1}=\lambda_{\theta_{1}} \vartheta_{s_{1}} \psi_{1}-\sigma_{\theta_{1}} \hat{\theta}_{1} . \\
u=N\left(\xi_{2}\right) \cdot \eta_{2} \\
\dot{\xi}_{2}=\vartheta_{s_{2}} \cdot \eta_{2} \\
\eta_{2}=\frac{K_{2} \sin \left(\frac{\pi S_{2}^{2}}{k_{b_{2}}^{2}}\right)}{2 S_{2}}+\hat{\theta}_{2} \psi_{2}+\frac{Z_{2}}{\tau_{2}} \\
\dot{\hat{\theta}}_{2}=\lambda_{\theta_{2}} \vartheta_{s_{2}} \psi_{2}-\sigma_{\theta_{2}} \hat{\theta}_{2} .
\end{gathered}
$$

where the even Nussbaum-type function is selected as $N\left(\xi_{i}\right)=\xi_{i}^{2} \cos (\xi)$.
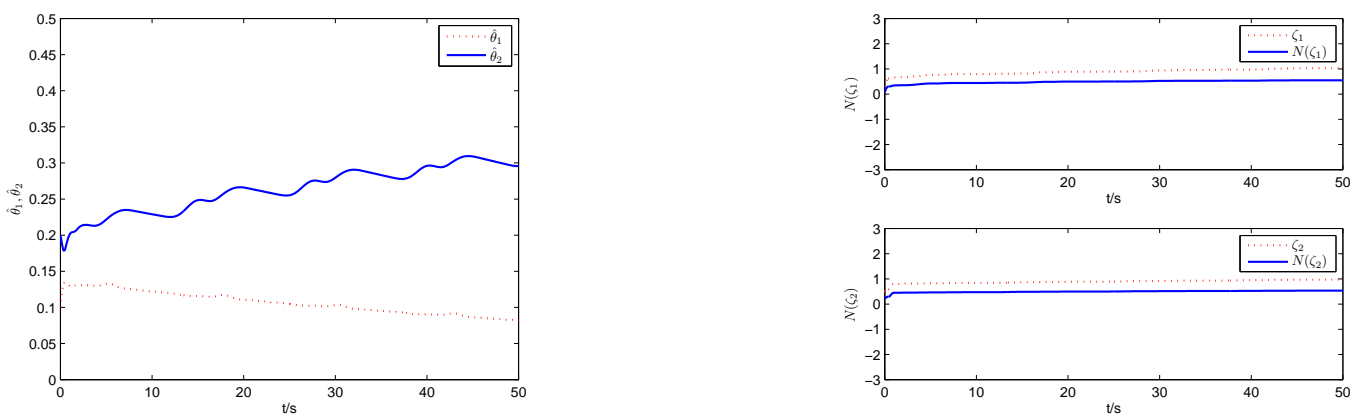

Figure 3: Trajectories of uncertain parameters(case one). Figure 4: Trajectories of the Nussbaum gain functions(case one).

Case two: $g_{1}=-1-\sin \left(x_{1}\right), g_{2}=1.5+\sin \left(x_{1} x_{2}\right) ; D_{1}=D_{2}=0$.

In this case we are talking about the case where there is no external disturbance, but the signs of the control gains $g_{1}$ and $g_{2}$ are opposite. Based on the same design parameters and initial values with case one, 


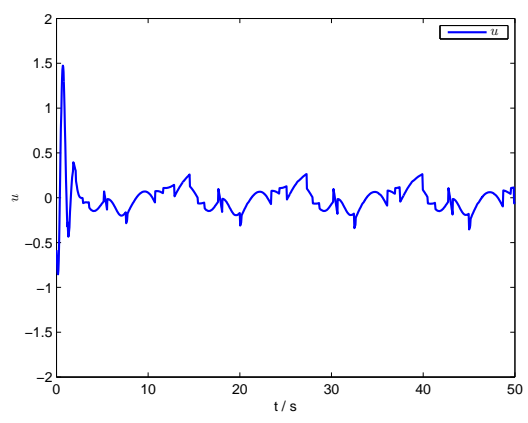

Figure 5: Trajectory of the input signal(case one).

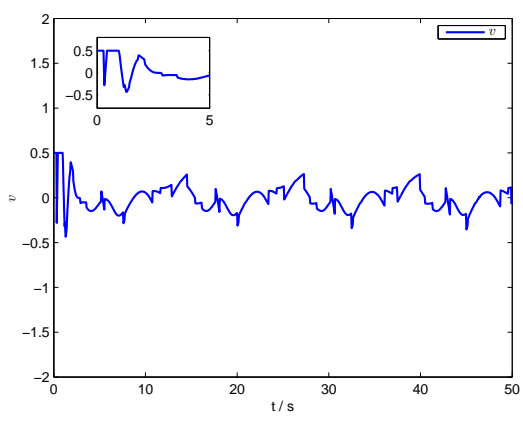

Figure 6: Trajectory of the saturated input signal(case one).

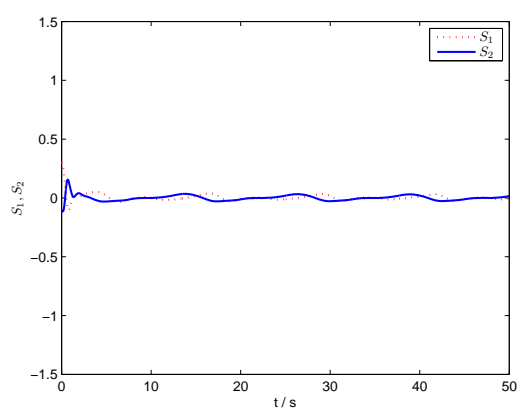

Figure 7: Trajectories of the errors(case one).

the simulation results are shown in Fig. 8-14.
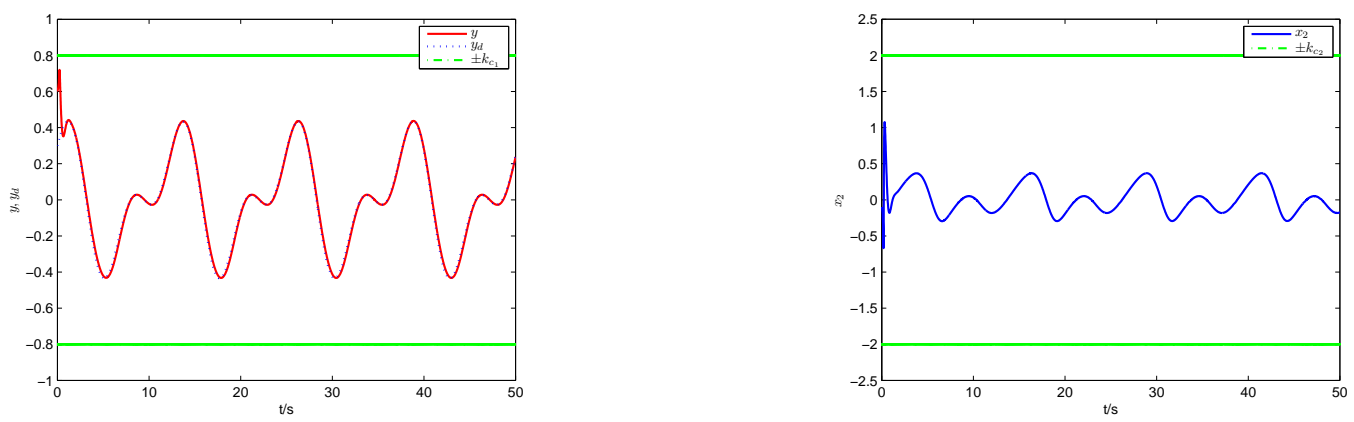

Figure 8: The trajectories of $y, y_{d}$ and their constraint(caseFigure 9: Trajectories of the state and its constraint(case two). two).

Remark 8. In this case, the signs of the control gain functions $g_{1}$ and $g_{2}$ are opposite. The simulation results demonstrate the crucial role of the Nussbaum function in the controller design. Nussbaum function can effectively adjust the unknown control direction. No matter the control direction is positive or negative, the controller we designed has a very good performance. By comparing the tracking errors of the two cases from Fig. 7 and Fig. 14, it can be seen that Fig. 14 only has a significant error within the first 1 second of operation due to the change of control direction. Then the controller quickly adjusted to achieve the similar tracking performance as the first case. From Fig. 12 and 13, it can be seen that the oscillation of the control input is 

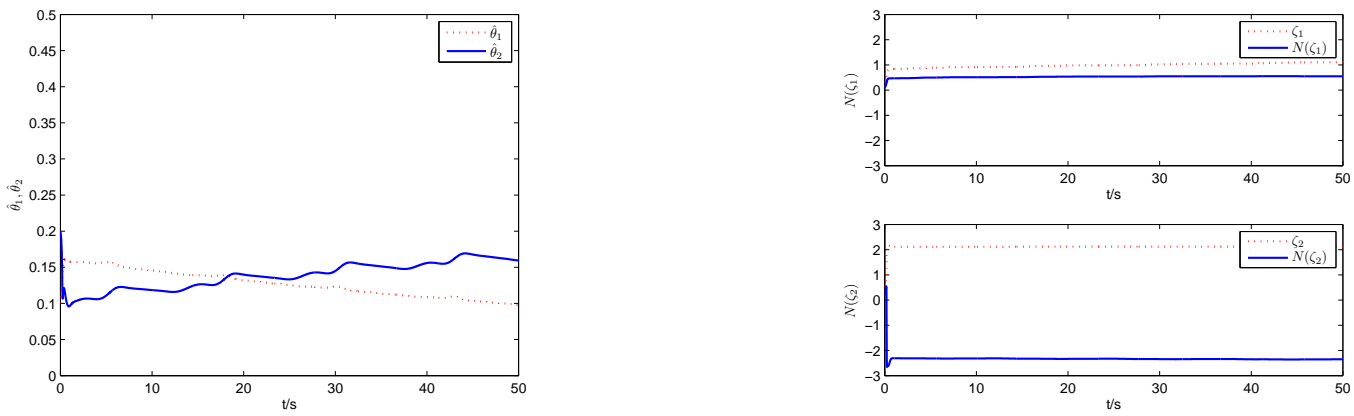

Figure 10: Trajectories of uncertain parameters(case two). Figure 11: Trajectories of the Nussbaum gain functions(case two).

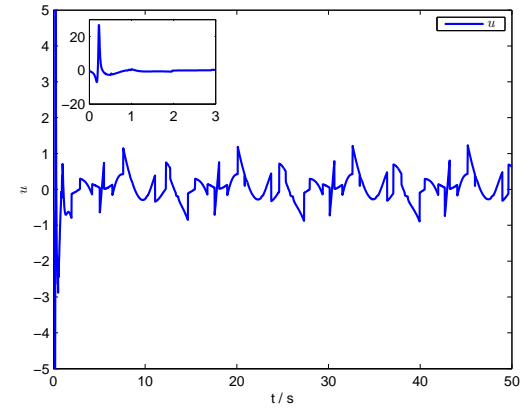

Figure 12: Trajectory of the input signal(case two).

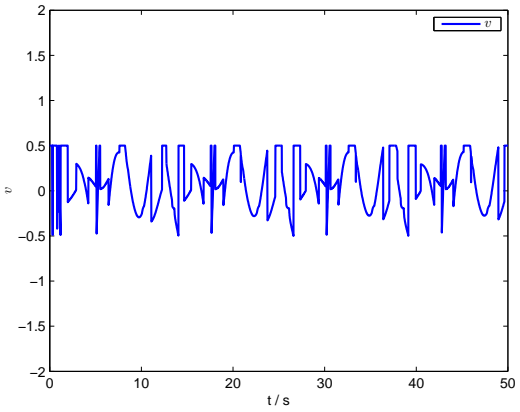

Figure 13: Trajectory of the saturated input signal(case two). 


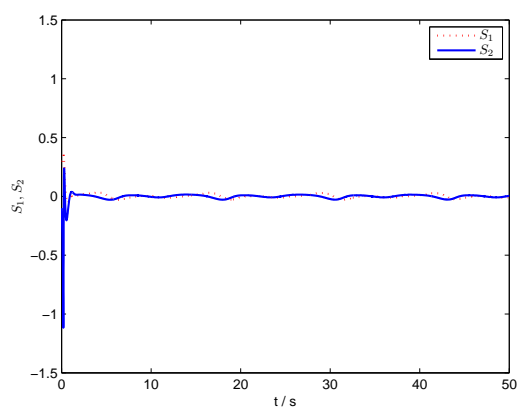

Figure 14: Trajectories of the errors(case two).

more obvious because of the change of control direction. Nevertheless, all of our preset control requirements are met.

Case three: $g_{1}=-1-\sin \left(x_{1}\right), g_{2}=1.5+\sin \left(x_{1} x_{2}\right) ; D_{1}=0.05 \sin (t), D_{2}=0.1 \sin (t)$.

In this case we are talking about the case where there exist unknown external disturbance, and the signs of the control gains $g_{1}$ and $g_{2}$ are opposite. Based on the same design parameters and initial values with above two cases, the simulation results are shown in Fig. 15-22.

Remark 9. In this case, different control direction and unknown external disturbance are considered. The simulation results illustrate the effectiveness of the proposed control scheme even in the presence of external interference. It can be seen from Fig. 15 and Fig. 22 that our tracking performance is better than the previous two cases in the case of external disturbance. That's because in the first two cases, where there is no external disturbance, we have do nothing with the redundant terms. In the third case, because of the disturbance, we combine the unknown bounds of the disturbance with other redundancies and design the adaptive law for them. It is not only eliminates the influence of the external disturbance, but also addresses other redundancy issues. That is the main reason for the better control performance.
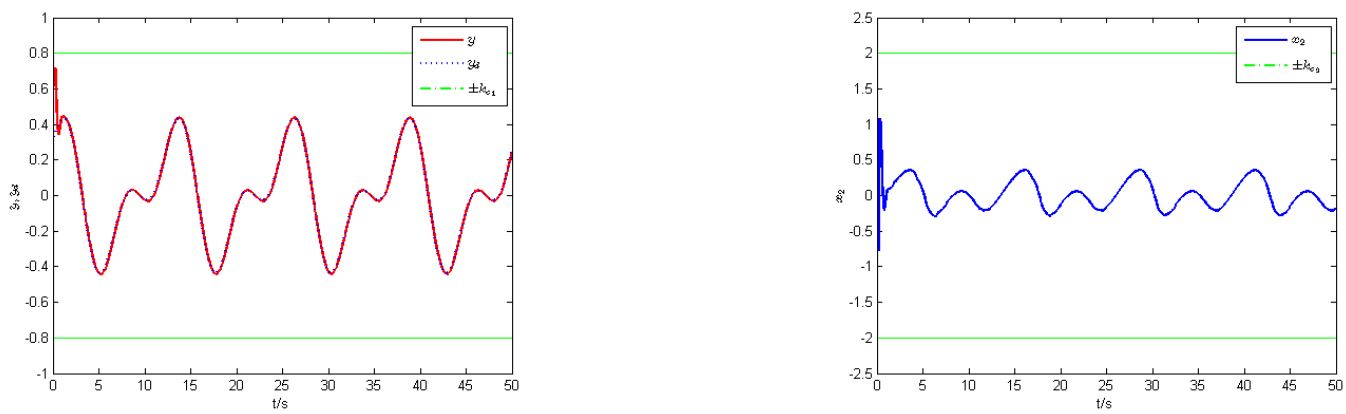

Figure 15: The trajectories of $y, y_{d}$ and their constrain-Figure 16: Trajectories of the state and its constraint(case $\mathrm{t}($ case three). three).

\section{Conclusions}

In this paper, a novel adaptive tracking control scheme is proposed for a class of nonlinear systems with full state constraints, input saturation, unknown control direction, and external disturbances. Using a hyperbolic 


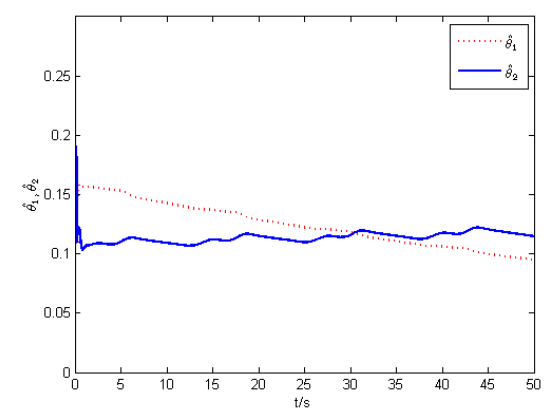

Figure 17: Trajectories of uncertain parameters(caseFigure three).
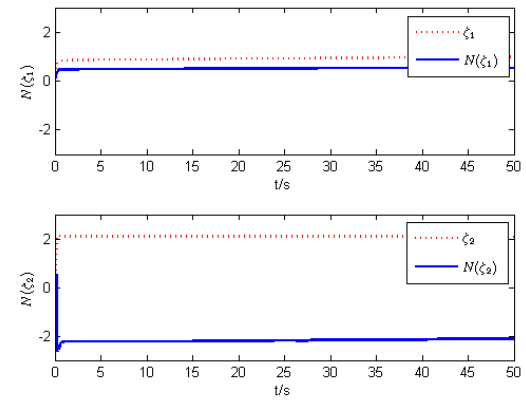

Figure 19: Trajectories of the Nussbaum gain functions(case three).

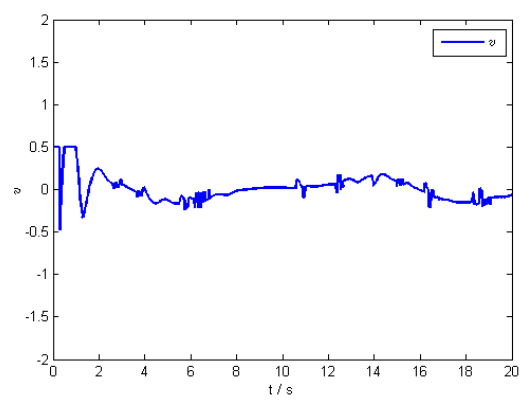

Figure 21: Trajectory of the saturated input signal(case three).

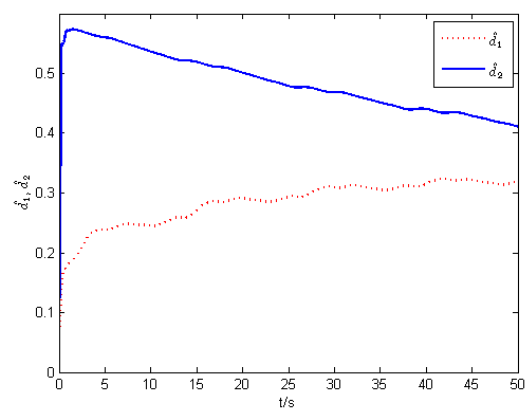

18: Trajectories of external disturbances(case three).

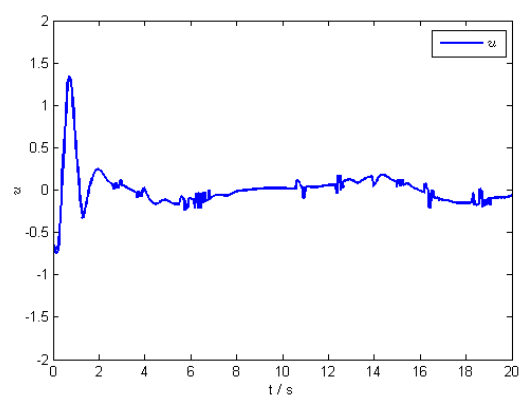

Figure 20: Trajectory of the input signal(case three).

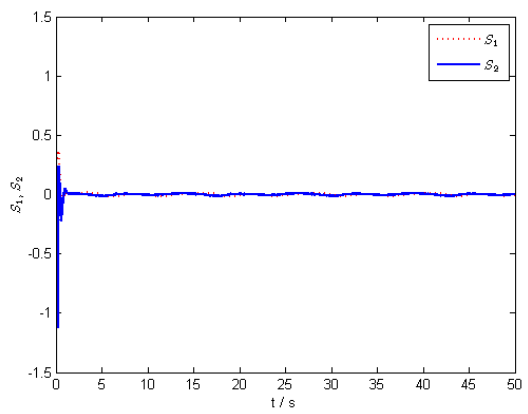

Figure 22: Trajectories of the errors(case three). 
tangent function to approximate the saturated input function, which avoids the sharp corner caused by the input function. The unknown control directions of system are solved by the Nussbaum-gain technique. And the state constraints are maintained by the involving of BLF. Meanwhile, dynamic surface control (DSC) is used in the controller design, which solved the problem of computation explosion caused by the repeated derivation of virtual control law. The proposed control strategy not only solves the problem of unknown control direction, the states are always within the constrained regions, but also makes the output tracking error of the system converges to a small neighborhood of zero closely. In future work, the control methods proposed in this paper can be further extended, such as systems with asymmetric time-varying state constraints, nonlinear systems with hysteresis, event-triggered constrained nonlinear systems, etc.

\section{Acknowledgements}

This work was supported in part by the National Natural Science Foundation of China under Grant No. 61803228, 61803229, and 62073187; Taishan Scholar Project of Shandong Province of China under Grant No. 2015162 and tsqn201812093; the Shandong Provincial Natural Science Foundation of China under Grant No. ZR2020QA036.

\section{Data availability statements}

The authors can confirm that all relevant data are included in the article.

\section{Conflict of interest}

The authors declare that they have no conflict of interest.

\section{References}

[1] M. Krstic and P. V. Kokotovic, Nonlinear Adaptive Control Design. New York: Wiley and Sons, 1995.

[2] M. Chen, S. S. Ge, and B. Ren, "Adaptive tracking control of uncertain MIMO nonlinear systems with input constraints," Automatica, vol. 47, no. 3, pp. 452-465, Mar. 2011.

[3] J.K. Ni, L. Liu, W. He, and C.X. Liu, "Adaptive dynamic surface neural network control for nonstrictfeedback uncertain nonlinear systems with constraints," Nonlinear Dyn., vol. 94, pp. 165-184.

[4] J. Du, X. Hu, and Y. Sun, "Adaptive robust nonlinear control design for course tracking of ships subject to external disturbances and input saturation," IEEE Trans. Systems, Man, and Cybernetics: Systems, vol. 50, no. 1, pp. 193-202, Jan. 2020.

[5] W. Qi, G. Zong, and H.R. Karimi, “Observer-based adaptive SMC for nonlinear uncertain singular semiMarkov jump systems with applications to DC motor," IEEE Trans. Circuits and Systems I: Regular Papers, vol. 65, no. 9, pp. 2951-2960, 2018. 
[6] W. He, S. S. Ge, B. V. E. How, Y. S. Choo, and K. S. Hong, "Robust adaptive boundary control of a flexible marine riser with vessel dynamics," Automatica, vol. 47, no. 4, pp. 722-732, 2011.

[7] W. Lin and C. Qian "Adaptive control of nonlinearly parameterized systems: A nonsmooth feedback framework," IEEE Trans. Automatic Control, vol. 47, no. 6, pp. 757-774, 2002.

[8] C.J. Xi, J.X. Dong, "Adaptive neural network-based control of uncertain nonlinear systems with timevarying full-state constraints and input constraint," Neurocomputing, vol. 357, pp.108-115, 2019.

[9] W.M. Chang, and S.C. Tong, "Adaptive fuzzy tracking control design for permanent magnet synchronous motors with output constraint," Nonlinear Dyn., vol. 87, pp. 291-302, 2017.

[10] Y.G. Yao, J.Q. Tan, J. Wu, and X. Zhang, "Event-triggered fixed-time adaptive fuzzy control for stateconstrained stochastic nonlinear systems without feasibility conditions," Nonlinear Dyn., vol. 105, pp. 403-416.

[11] C. Chen, Z. Liu, Y. Zhang, C. L. P. Chen, and S. Xie, "Saturated Nussbaum function based approach for robotic systems with unknown actuator dynamics," IEEE Trans. Cybern., vol. 46, no. 10, pp. 2311-2322, Oct. 2016.

[12] J. L. Du, C. Guo, S. H. Yu, and Y. S. Zhao, "Adaptive autopilot design of time-varying uncertain ships with completely unknown control coefficient, " IEEE Journal of Oceanic Engineering, vol. 32, no. 2, pp. 346-352, 2007.

[13] R. D. Nussbaum, "Some remarks on a conjecture in parameter adaptive control," Syst. Control Lett., vol. 3, no. 5, pp. 243-246, 1983.

[14] H. Ma, H. J. Liang, Q. Zhou, and C. K. Ahn, "Adaptive dynamic surface control design for uncertain nonlinear strict-feedback systems with unknown control direction and disturbances," IEEE Trans. Systems, Man, and Cybernetics: Systems, vol. 49, no. 3, pp. 506-515, Mar. 2019.

[15] P. Wang, C. P. Yu, and J. Sun, "Global output feedback control for nonlinear cascade systems with unknown output functions and unknown control directions," Int J Robust Nonlin Control, vol. 30, no. 6, pp. 2493-2514, Apr. 2020.

[16] W. Sun, J.W. Xia, G.M. Zhuang, X. Huang, and H. Shen, "Adaptive fuzzy asymptotically tracking control of full state constrained nonlinear system based on a novel nussbaum-type function," J. Frankl. Inst., vol. 356, no. 4, pp. 1810-1827, 2019.

[17] A. Kamalamiri, M. Shahrokhi, and M. Mohit, "Adaptive finite-time neural control of non-strict feedback systems subject to output constraint, unknown control direction, and input nonlinearities," Information Sciences, vol. 520, pp. 271-291, 2020.

[18] S.S. Ge, J. Wang, "Robust adaptive tracking for time-varying uncertain nonlinear systems with unknown control coefficients," IEEE Trans. Automat. Contr., vol. 48, pp. 1463-1469, 2003.

[19] K. P. Tee, S. S. Ge, and E. H. Tay, "Adaptive control of electrostatic microactuators with bidirectional drive," IEEE Trans. Control Systems Technology, vol. 17, no. 2, pp. 340-352, Feb. 2009. 
[20] W. He, Y. Chen, and Z. Yin, "Adaptive neural network control of an uncertain robot with full-state constraints," IEEE Trans. Cybernetics, vol. 46, no. 3, pp. 620-629, Mar. 2016.

[21] A. Bemporad, "Reference governor for constrained nonlinear systems," IEEE Transactions on Automatic Control, vol. 43, no. 3, pp. 415-419, Mar. 1998.

[22] F. Blanchini, "Set invariance in control," Automatica, vol. 35, no. 11, pp. 1747-1767, Nov. 1999.

[23] D. Q. Mayne, J. B. Rawlings, C. V. Rao, and P. O. M. Scokaert, "Constrained model predictive control: stability and optimality," Automatica, vol. 36, no. 6, pp. 789-814, Jun. 2000.

[24] K. B. Ngo, R. Mahony, and Z. P. Jiang, "Integrator backstepping using barrier functions for systems with multiple state constraints," in: Proc. of the 44rd Conf. Decision and control, Seville, Spain, Dec. 12-15, 2005, pp. 8306-8312.

[25] K. P. Tee, S. S. Ge, and E. H. Tay, "Barrier Lyapunov functions for the control of output-constrained nonlinear systems," Automatica, vol. 45, no. 4, pp. 918-927, Apr. 2009.

[26] Y. J. Liu, S. M. Lu, D. J. Li, and S. C. Tong, "Adaptive controller design-based ABLF for a class of nonlinear time-varying state constraint systems," IEEE Trans. Systems, Man, and Cybernetics: Systems, vol. 47, no. 7, pp. 1546-1553, Jun. 2017.

[27] C. X. Wang, Y. Q. Wu, and J. B. Yu, "Barrier Lyapunov functions-based dynamic surface control for pure-feedback systems with full state constraints," IET, Control Theory and Applications, vol. 11, no. 4, pp. 524-530, Apr. 2017.

[28] C. X. Wang, J. L. Du, and J. B. Yu, "Adaptive finite-time tracking control for timevarying output constrained non-linear systems with unmatched uncertainties," IET, Control Theory and Applications, vol. 13, no. 15, pp. 2416-2424, 2019.

[29] C. X. Wang, X. F. Liu, J. B. Yu, and J. L. Yu, "State transformation-based adaptive tracking control for a class of nonlinear systems with time-varying state constraints," ISA Transactions, vol. 110, pp.129-137, Mar. 2021.

[30] B. Niu, D. Wang, H. Li, X. Xie, N. D. Alotaibi, and F. E. Alsaadi, "A novel neural-network-based adaptive control scheme for output-constrained stochastic switched nonlinear systems," IEEE Transactions on Systems, Man, and Cybernetics: Systems, vol. 49, no. 2, pp. 418-432, Feb. 2019.

[31] K. Zhao and Y. D. Song, "Removing the feasibility conditions imposed on tracking control designs for state-constrained strict-feedback systems," IEEE Trans. Automatic Control, vol. 64, no. 3, pp. 12651272, Mar. 2019.

[32] Y. H. Kim and F. L. Lewis, "Reinforcement adaptive control of a class of neural-net-based friction compensation control for high speed and precision," IEEE Trans. Control Syst. Technol., vol. 8, no. 1, pp. 118-126, Jan. 2000. 
[33] A. Leonessa, W. M. Haddad, T. Hayakawa, and Y. Morel, "Adaptive control for nonlinear uncertain systems with actuator amplitude and rate saturation constraints," Int. J. Adaptive Control Signal Processing, vol. 23, no. 1, pp. 73-96, 2009.

[34] E. Lavretsky and N. Hovakimyan, "Positive $\mu$-modification for stable adaptation in dynamic inversion based adaptive control with input saturation," in Proc. Amer. Control Conf., 2005, pp. 3373-3378.

[35] Y. Yang, J. Tan , and D. Yue. "Prescribed performance tracking control of a class of uncertain purefeedback nonlinear systems with input saturation," IEEE Trans. Systems, Man, and Cybernetics: Systems, vol. 50, no. 5, pp. 1733-1745, May 2020.

[36] C. Wen, J. Zhou, Z. T. Liu, and H. Su, "Robust adaptive control of uncertain nonlinear systems in the presence of input saturation and external disturbance," IEEE Trans. Automat. Contr., vol. 56, no. 7, pp. 1672-1678, 2011.

[37] K. P. Tee, S. S. Ge, "Control of nonlinear systems with partial state constraints using a barrier Lyapunov function,” Int. J. Control, vol. 84, no. 12, pp. 2008-2023, 2011. 